\title{
Neglect Benevolence in Human Control of Robotic Swarms
}

\author{
Sasanka Nagavalli, Student Member, IEEE, Lingzhi Luo, Student Member, IEEE, \\ Nilanjan Chakraborty, Member, IEEE, Katia Sycara, Fellow, IEEE
}

\begin{abstract}
Robotic swarms are distributed systems whose members interact via local control laws to achieve different behaviors. Practical missions may require a combination of different swarm behaviors, where these behavioral combinations are not known a priori but could arise dynamically due to changes in mission goals. Therefore, human interaction with the swarm (HIS) is needed. In this paper, we introduce, formally define and characterize a novel concept, Neglect Benevolence, that captures the idea that it may be beneficial for system performance if the human operator, after giving a command, waits for some time before giving a subsequent command to the swarm. This raises the important question of the existence and means of calculation of the optimal time for the operator to give input to the swarm in order to optimize swarm behavior. Human operators are limited in their ability to estimate the best time to give input to the swarm. Therefore, automated aids that calculate the optimal input time could help the human operator achieve the best system performance. Our contributions are as follows. First, we formally define the new notion of Neglect Benevolence. Second, we prove the existence of Neglect Benevolence for a class of linear dynamical systems. Third, we provide an analytic characterization and an algorithm for calculating the optimal input time. Fourth, we apply the analysis to the human control of swarm configuration.
\end{abstract}

\section{INTRODUCTION}

Swarm robotic systems use control laws based on local spatial information about the environment and/or other members of the swarm within their spatial neighborhood to achieve various collective behaviors. The key advantage of swarms is that the behaviors generated are robust to individual robot failures. Practical missions or tasks for robotic systems may require a combination of different swarm behaviors in dynamic environments or situations. However, swarms are not yet capable of performing such complex dynamic missions autonomously. Human experiments with a simulated swarm system performing a variety of tasks including environment exploration [1], radiation source detection [2] have been performed. In a previous experimental study in a foraging scenario [3], we found that the performance of the system was affected by the time between two commands that the human was applying to the robots. In particular, we found that one group of subjects who performed well waited for some time after they issued a command before issuing another corrective command (when they wanted to change the direction in which the swarm was heading). We termed the phenomenon Neglect Benevolence,

The authors are with the Robotics Institute, School of Computer Science, Carnegie Mellon University, Pittsburgh, Pennsylvania, USA. Email: \{snagaval, lingzhil\}@andrew.cmu.edu, \{nilanjan, katia\}@cs.cmu.edu.

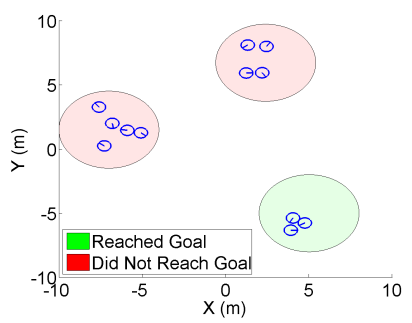

(a) When the human input is applied at $t=0$ the swarm split and only 3 robots reach the goal

Fig. 1: Effect of Different Human Control Input Times on the Final State of the Swarm

since neglecting the swarm (or not "correcting" the swarm) for some amount of time leads to better performance of the system. The goal of this paper is to formalize the notion of Neglect Benevolence.

Our human experiment findings indicated that when the swarm was in a transient state (i.e. moving towards one goal), application of another input (that changes the goal) could have different effects depending on the timing of the inputs. To verify this in a more controlled setting we set up a simulation of a small swarm system (12 robots) performing rendezvous. The robots move under simple control laws given in [4] with a repulsive potential to avoid inter-robot collision and an attractive potential field to maintain swarm cohesion. The operator inputs change the point at which the agents rendezvous. Figure 1 shows an example of the difference in the end result when the input is applied at two different times. In Figure 1a when the input is applied immediately after the operator wants to change the goal point of the rendezvous, the robot swarm splits and some of the robots, shown in the figure via the pink circles, wander off never reaching the operator goal, and only a small number (3) of robots, shown in the green circle, reaches the goal. However, when there is a delay in applying the input, shown in Figure 1b, the swarm stays as a single entity and converges around the operator goal. This example further reinforces the experimental findings that for a human to influence the operations of a robotic swarm the timing of the human input can greatly affect the performance of the robotic system. We performed additional simulations with large numbers of robots under different control laws and initial configurations and found similar results in that the timing of the operator input affected the system performance.

Since the behavior of a swarm system is emergent via 
the robot autonomous interactions, it is difficult for humans to have an intuitive understanding of the evolution of the swarm states. Consequently, it is difficult for humans to have a reasonable guess as to the appropriate delay after which to apply the input. Therefore, formal characterization of the notion of Neglect Benevolence and methods to calculate the best time the operator must give an input are needed. These results would also be of practical significance since they would enable the design of computational aids for the human to ensure that the human input is given to the swarm at the appropriate time.

Note that for the results shown in Figure 1, the underlying dynamics of the swarm system is nonlinear and the results show that the inappropriate timing of the human input can make the system unstable. However, the system does not need to be nonlinear for Neglect Benevolence to be present. Even if (a) the system dynamics is linear and (b) the swarm is always guaranteed to be stable, which is the case with well-designed linear systems, Neglect Benevolence can still exist (as we will show later in the paper).

We first introduce a formal definition of Neglect Benevolence. Different performance measures for the swarm system can be used for defining neglect benevolence. Examples include the time taken by the swarm to reach the human goal and the energy used by the swarm to reach the goal. We will then study a particular class of linear systems, namely linear systems with normal dynamics matrix where the performance measure is defined as the time taken by the swarm to reach the human specified goal. The dynamics matrix of a swarm that perform consensus falls within this class. The human input can control the inter-robot spacing and thereby control the final shape into which the robots converge. We will illustrate our concepts with this example (see Section V). Our paper makes the following contributions: (a) We formally define the new notion of Neglect Benevolence, (b) We prove the existence of Neglect Benevolence for a set of linear dynamical systems, (c) We provide an analytic characterization and an algorithm for calculating the optimal input time, (d) We apply the analysis to the human control of swarm configuration to illustrate the approach.

This paper is organized as follows: In Section II we present a discussion of the related work. In Section III we present a formal notion of Neglect Benevolence and prove the existence of Neglect Benevolence for a class of linear systems. In Section IV we present a procedure to compute the delay time for linear systems with normal dynamics matrix. In Section $\mathrm{V}$ we show the application of the analysis for configuration control of swarms. Thereafter, in Section VI, we present our simulation results and in Section VII we present our conclusions and outline avenues for future work.

\section{RELATED WORK}

Robotic swarm systems where a group of robots need to act through local interactions to collectively achieve a variety of behaviors have been extensively studied. Swarms can achieve behaviors such as flocking [5]-[8], rendezvous [9], deployment [10], and foraging [2], [11]. Formation control of robotic swarms has been proposed to adjust the spatial configuration of robotic systems so that they satisfy the requirements of deployment tasks or they can adapt to different environments while performing tasks [12]-[15].

However, schemes which include human operator in the loop together with robotic swarms are required for some complicated tasks (e.g. complex surveillance and reconnaissance). For using swarm robotic systems in humansupervised missions, human swarm interaction (HSI) has been studied in the extant literature [1], [16]-[20]. The concept of Neglect Tolerance has been introduced to capture the idea that a human operator can neglect robots that work independently of one another in a multi-robot system for a certain time before system performance degrades [21][24]. One key issue in neglect tolerance is how to schedule the attention of the human operator among the multiple and independent robots so that the neglect time between servicing robots is minimized [25]-[28]. In Neglect Tolerance, it is assumed that the robot system's performance will degrade over time and the system will need human interaction to restore performance to a desirable level. In contrast, the concept of Neglect Benevolence is proposed in human swarm interaction to capture the idea that it may be beneficial for the human operators to wait for a certain length of time before applying input to the system while the swarm state evolves to stabilization (since the swarm state may not degrade monotonically with time) [3]. The concept of Neglect Benevolence has not been quantitatively characterized to date. Based on the observation of Neglect Benevolence in human control of robotic swarms, we propose a formal way to characterize Neglect Benevolence by considering the system dynamics and under different performance criteria.

\section{Formalizing the Notion of NeGlect BENEVOLENCE}

Consider a system that consists of a swarm of robots guided by a human operator. The robot swarm can be modeled as an autonomous distributed dynamical system that is capable of incorporating an input from an intelligent external controller (i.e. human operator). Each individual component of the distributed dynamical system has a state $\mathbf{x}^{(i)}$ that evolves as a function of time $t$. For a system with $m$ components, the joint state $\mathbf{x}$ of the entire dynamical system (i.e. swarm) is given by concatenating the states of the individual components (i.e. robots). The set of all states of the system is known as the state space $\mathcal{X}$. The state $\mathrm{x} \in \mathcal{X}$ of the entire system evolves over time according to the inherent system dynamics $f$, which relate the change in the state over time $\dot{\mathrm{x}}$ to the current state $\mathbf{x}$ and autonomous input $\mathbf{u}_{a} \in \mathcal{U}_{a}$, where $\mathcal{U}_{a}$ is a bounded set. Each robot in the system contains an automatic controller $g^{(i)}$ that computes the autonomous input $\mathbf{u}_{a}^{(i)}$ to the robot's actuators based on an estimate $\hat{\mathbf{x}}$ of the state of the system and the human input $\mathbf{u}_{h}$. The human has a desired - possibly time-varying — set of goals that he/she would like to achieve with the robot swarm and a fixed set of inputs $\mathcal{U}_{h}$ that they can apply to influence the swarm. The goals may arise dynamically due to unexpected 
events in the environment or mission changes and are not known in advance. The goals can be expressed as a set of goal states $\mathcal{X}_{g} \subset \mathcal{X}$. The human combines this knowledge of desired goal states $\mathcal{X}_{g}$ with their possibly noisy, incorrect or incomplete knowledge $\tilde{\mathbf{x}}$ of the swarm's current state via a process $h$ to generate an input $\mathbf{u}_{h} \in \mathcal{U}_{h}$ to the robot swarm. The relationships between all of these variables are summarized in the equations below.

$$
\begin{aligned}
& \mathbf{x}(t)=\left[\begin{array}{llll}
\mathbf{x}^{(1)}(t)^{T} & \mathbf{x}^{(2)}(t)^{T} & \cdots & \mathbf{x}^{(m)}(t)^{T}
\end{array}\right]^{T} \\
& \mathbf{u}_{a}(t)=\left[\begin{array}{llll}
\mathbf{u}_{a}^{(1)}(t)^{T} & \mathbf{u}_{a}^{(2)}(t)^{T} & \cdots & \mathbf{u}_{a}^{(m)}(t)^{T}
\end{array}\right]^{T} \\
& \dot{\mathbf{x}}(t)=f\left(\mathbf{x}(t), \mathbf{u}_{a}(t)\right) \\
& \mathbf{u}_{a}^{(i)}(t)=g^{(i)}\left(\hat{\mathbf{x}}(t), \mathbf{u}_{h}(t)\right) \\
& \mathbf{u}_{h}(t)=h\left(\tilde{\mathbf{x}}(t), \mathcal{X}_{g}\right)
\end{aligned}
$$

For the purposes of this paper, we will just assume that the human can come up with the input $\mathbf{u}_{h}$ without modeling the function $h$. Furthermore, we will assume that each robot in the swarm has perfect access to its own and its neighbors' state (i.e. $\hat{\mathbf{x}}=\mathbf{x}$ ). The set of stable equilibrium points $\mathcal{X}_{n} \subset$ $\mathcal{X}$ of the system is called the natural goal set. When there is no human influence (i.e. $\mathbf{u}_{h}=\mathbf{0}$ ), any control input $\mathbf{u}_{n} \in$ $\mathcal{U}_{a}$ that drives the system to the natural goal set is called a natural input.

A path through the state space is a sequence of states that begins at some state $\mathbf{x}_{s}$ at $t=0$ and approaches $\mathbf{x}_{e}$ as $t \rightarrow \infty$. The path taken by a system from any initial state $\mathbf{x}_{s}$ towards a natural goal, when $\mathbf{u}_{h}=\mathbf{0}$, is called the natural path.

As the system proceeds towards its natural goal $\mathbf{x}_{n}$, the human may wish to apply an input $\mathbf{u}_{h}$ to influence the swarm towards the desired set of goal states $\mathcal{X}_{g}$. The input may be instantaneous or persistent, but for illustration, assume that there is only one opportunity to apply or activate it. Given a performance criterion that the human wishes to maximize, if applying the input at some time $T>0$ results in better performance than applying the input at any time $t<T$, then all states of the system during time $t<T$ are said to be Neglect Benevolent. Given a system, a performance criterion, an input $\mathbf{u}_{h} \in \mathcal{U}_{h}$ and a set of desired goal states $\mathcal{X}_{g}$, there may exist a set of Neglect Benevolent states $\mathcal{X}_{n b} \subset$ $\mathcal{X}$ where delaying the application of the input will result in improved system performance. Specifically, when the system begins in state $\mathbf{x}_{n b} \in \mathcal{X}_{n b}$ and follows a natural path through the state space, there is some future state at which applying the input results in better performance than applying the input at state $\mathbf{x}_{n b}$. Systems where $\mathcal{X}_{n b} \neq \varnothing$ are said to exhibit the property of Neglect Benevolence. The performance criterion to be used is task-specific and depends on the mission goals. Examples of performance criteria include the time taken to reach the human goal and the total energy required to reach the human goal. For the rest of the paper, we will use the time required by the system to reach the human goal as the performance criterion.

\section{Analysis of Neglect BeneVolence in Linear TIME INVARIANT SYSTEMS}

In this section, we quantitatively analyze the concept of Neglect Benevolence in human control of robot swarm systems. In Section V, we use the analysis to determine Neglect Benevolence and best time to apply human input for swarm configuration control. We focus on linear time invariant (LTI) robot swarm systems with a normal dynamics matrix. In Section IV-A, we first consider a swarm system without external human input, and numerically solve the system convergence time based on the eigenvalue decomposition. Then we discuss the situation when the human operator wants to give a new command to the dynamic swarm system, and present an algorithmic way of finding the optimal time to impose the human operator's new input so that the time to reach the new goal is minimized. In Section V, we analyze an application of human control in robot swarm configuration control. We first design a control law for a robot swarm system so that it can form desired spatial configurations. The swarm system under the designed control law has a symmetric dynamics matrix, and we apply the above analysis results to optimize the configuration control of the system.

\section{A. Neglect Benevolence in LTI Systems with a Normal Dy- namics Matrix}

Consider a linear time-invariant (LTI) robot swarm system with a normal dynamics matrix and without external human inputs.

$$
\begin{aligned}
\dot{\mathbf{x}}(t) & =\mathbf{A x}(t) \\
\mathbf{x}(0) & =\mathbf{x}_{0}
\end{aligned}
$$

where $\mathbf{A}$ is the dynamics matrix, $\mathbf{x}(t)$ represents the system state vector at time $t$ and $\mathbf{x}_{0}$ is the initial state. We assume that $\mathbf{A}$ is normal (i.e. $\mathbf{A}^{T} \mathbf{A}=\mathbf{A} \mathbf{A}^{T}$ ) and all its eigenvalues have negative real parts (so that the system is stable). Below we first numerically solve the system convergence time based on the eigenvalues of $\mathbf{A}+\mathbf{A}^{T}$.

Solving the dynamic equation, we get

$$
\mathbf{x}(t)=e^{\mathbf{A} t} \mathbf{x}_{0}
$$

Since $\mathbf{A}$ is normal, $\mathbf{A}^{T} \mathbf{A}=\mathbf{A} \mathbf{A}^{T}$, so

$$
\|\mathbf{x}(t)\|_{2}^{2}=\mathbf{x}_{0}^{T} \mathbf{Q} e^{\mathbf{\Lambda} t} \mathbf{Q}^{T} \mathbf{x}_{0}
$$

where $\mathbf{A}^{T}+\mathbf{A}=\mathbf{Q} \mathbf{\Lambda} \mathbf{Q}^{T}$ is the eigenvalue decomposition of the symmetric matrix $\left(\mathbf{A}^{T}+\mathbf{A}\right)$ and $\boldsymbol{\Lambda}$ is a diagonal matrix with elements $\lambda_{i}$, where $\lambda_{i} \in \mathbb{R}$ are the eigenvalues of $\left(\mathbf{A}^{T}+\mathbf{A}\right)$. Define $\mathbf{y}=\mathbf{Q}^{T} \mathbf{x}_{0}$, we have

$$
\|\mathbf{x}(t)\|_{2}^{2}=\mathbf{y}^{T} e^{\boldsymbol{\Lambda} t} \mathbf{y}=\sum y_{i}^{2} e^{\lambda_{i} t}
$$

From the above, the 2-norm of the state evolution function is a sum of decaying exponentials with positive coefficients. We can find an upper bound on the convergence time $t_{\text {natural }}$ for the system without external input using the maximum 
eigenvalues. Define $\alpha=\max _{i} \lambda_{i}$ and $\varepsilon$ as the tolerance for convergence.

$$
\begin{aligned}
\left\|\mathbf{x}\left(t_{\text {natural }}\right)\right\|_{2}^{2} & =\sum y_{i}^{2} e^{\lambda_{i} t_{\text {natural }}} \leq \mathbf{y}^{T} \mathbf{y} e^{\alpha t_{\text {natural }}}<\varepsilon^{2} \\
t_{\text {natural }} & \leq \frac{1}{\alpha} \ln \frac{\varepsilon^{2}}{\mathbf{y}^{T} \mathbf{y}}=\frac{1}{\alpha} \ln \frac{\varepsilon^{2}}{\mathbf{x}_{0}^{T} \mathbf{x}_{0}}
\end{aligned}
$$

Define $f(t)=\left\|e^{\mathbf{A} t} \mathbf{x}_{0}\right\|_{2}^{2}$ and $g(t)=f(t)-\varepsilon$. Since $f(t)$ is monotonically decreasing and converges to $0, g(t)$ only has one root, which represents the convergence time of the system. A numerical solution for the convergence time $t_{\text {natural }}$ can be found using the following iterative Newton's Method.

$$
t_{k+1}=t_{k}-\frac{g(t)}{g^{\prime}(t)}
$$

where $g^{\prime}(t)=\sum_{i=1}^{n} y_{i}^{2} \lambda_{i} e^{\lambda_{i} t}$.

Now consider the situation when the human operator wants to give a new input to the dynamic swarm system.

$$
\dot{\mathbf{x}}(t)=\mathbf{A}\left(\mathbf{x}(t)-K \theta\left(t-t_{\text {input }}\right)\right)
$$

where $K$ represents the new input, so that the system now converges to state $K$ rather than the origin. Here $\theta(t)$ is the Heaviside step function such that $\theta(t)=0$ for $t<0$ and $\theta(t)=1$ for $t \geq 0$. $t_{\text {input }}$ is the time when the new input $K$ is imposed on the system's dynamics. Solving the dynamics equation, we have

$\mathbf{x}(t)=e^{\mathbf{A} t}\left(\mathbf{x}_{0}-e^{-\mathbf{A} t_{\text {input }}} K \theta\left(t-t_{\text {input }}\right)\right)+K \theta\left(t-t_{\text {input }}\right)$

For a stable system, $e^{\mathbf{A} t}$ is a decaying matrix exponential, so the system will converge to the input $K$ at some time $t_{\text {goal }}$ after $t_{\text {input }}$.

$$
\mathbf{x}\left(t_{\text {goal }}\right)=e^{\mathbf{A} t_{\text {goal }}}\left(\mathbf{x}_{0}-e^{-\mathbf{A} t_{\text {input }}} K\right)+K
$$

Define $\mathbf{x}_{d}=\mathbf{x}_{0}-e^{-\mathbf{A} t_{\text {input }}} K$,

$$
\begin{aligned}
\mathbf{x}\left(t_{\text {goal }}\right) & =e^{\mathbf{A} t_{\text {goal }}} \mathbf{x}_{d}+K \\
\left\|e^{\mathbf{A} t_{\text {goal }}} \mathbf{x}_{d}\right\|_{2} & <\varepsilon
\end{aligned}
$$

So we can solve $t_{\text {goal }}$ in the same way as solve $t_{\text {natural }}$ in the case without new input and the only difference is that the initial condition changes from $\mathbf{x}_{0}$ to $\mathbf{x}_{d}$.

The convergence time $t_{\text {goal }}$ depends on $t_{\text {input }}$ (implicitly included in the expression of $\mathbf{x}_{d}$ ). We can use the following naive algorithm to sample all possible $t_{\text {input }} \leq t_{\text {natural }}$ and find the best input time $t_{\text {input }}^{*}$ so that $t_{\text {goal }}$ is minimized:

1) Solve the dynamics equations (i.e. find $\mathbf{x}(t)$ ).

2) Find the convergence time to the natural goal without external input.

$$
t_{\text {natural }}=\min \left\{t_{f} \mid \forall t \geqslant t_{f}:\left\|e^{\mathbf{A} t} \mathbf{x}_{0}\right\|_{2}<\varepsilon\right\}
$$

$3)$ For each possible input time $t_{\text {input }} \leqslant t_{\text {natural }}$, compute the convergence time to the new input goal.

$t_{\text {goal }}=\min \left\{t_{f} \mid \forall t \geq t_{f}:\left\|e^{\mathbf{A} t}\left(\mathbf{x}_{0}-e^{-\mathbf{A} t_{\text {input }}} K\right)\right\|_{2}<\varepsilon\right\}$
4) The input time, $t_{\text {input }}^{*}$, that results in the minimum convergence time to the new input goal, $t_{\text {goal }}$, is the optimal input time.

Whenever $t_{\text {input }}^{*}>0$, it means that the new input should be delayed for $t_{\text {input }}^{*}$ before imposed on the system, so that the convergence performance of the system is optimized. Generally speaking, whether $t_{\text {input }}^{*}=0$ or $t_{\text {input }}^{*}>0$ would depend on the dynamics matrix $\mathbf{A}$, the natural goal, the newly input goal $K$, as well as the initial state $\mathbf{x}_{0}$. However, for any dynamics matrix $\mathbf{A}$ (normal and exponentially stable), we can construct a condition (including the new goal $K$, initial state $\mathbf{x}_{0}$ ), such that under such a condition, $\mathbf{x}_{0}$ is always Neglect Benevolent, and thus $t_{\text {input }}^{*}>0$ (See Theorem 1). The above analysis provides a quantitative way to characterize Neglect Benevolence.

Theorem 1: Consider an exponentially stable linear system where the human input is incorporated using Equation 12.

$$
\dot{\mathbf{x}}(t)=\mathbf{A}\left(\mathbf{x}(t)-K \theta\left(t-t_{\text {input }}\right)\right)
$$

In such a system, for any initial state $\mathbf{x}_{0}$, if the state $K$ lies along the natural path from $\mathbf{x}_{0}$ to the origin, then the state $\mathbf{x}_{0}$ must be Neglect Benevolent.

Proof: Let the system take time $t_{1}$ to go from initial state $\mathbf{x}_{0}$ to the state $K$ along the natural path under the dynamics $\dot{\mathbf{x}}(t)=\mathbf{A} \mathbf{x}(t)$. Without loss of generality, we can assume that there is a state $\mathbf{x}_{1}$ along the natural path such that the time taken to goal from state $K$ to state $\mathbf{x}_{1}$ is $t_{1}$. Define $\mathbf{x}_{2}=K-\mathbf{x}_{1}$. Suppose that it takes time $t_{2}$ from $\mathbf{x}_{0}$ to $K$ under the new input dynamics $\dot{\mathbf{x}}(t)=\mathbf{A}(\mathbf{x}(t)-K)$.

$$
\begin{array}{r}
e^{\mathbf{A} t_{1}} \mathbf{x}_{0}=K \\
\left\|e^{\mathbf{A} t_{2}}\left(\mathbf{x}_{0}-K\right)\right\|_{2}<\varepsilon
\end{array}
$$

Substituting Equation 13 to Equation 14, we have

$$
\begin{array}{r}
\left\|e^{\mathbf{A}\left(t_{2}-t_{1}\right)}\left(I-e^{\mathbf{A} t_{1}}\right) K\right\|_{2}<\varepsilon \\
\left\|e^{\mathbf{A}\left(t_{2}-t_{1}\right)} \mathbf{x}_{2}\right\|_{2}<\varepsilon
\end{array}
$$

Thus, the time taken to go from state $\mathbf{x}_{2}$ to the origin along the natural path is $t_{2}-t_{1}$, which must be positive. So $t_{2}>t_{1}$, which means that it takes longer when $t_{\text {input }}=0$ than $t_{\text {input }}=t_{1}$ for the system to reach the state $K$ from initial state $\mathbf{x}_{0}$. So $\mathbf{x}_{0}$ must be Neglect Benevolent.

In Theorem 1, we showed that for any input $K$, we can find at least one initial state $\mathbf{x}_{0}$ that must be Neglect Benevolent. Conversely, it can be shown via similar arguments that given any initial state $\mathbf{x}_{0}$, we can find at least one input $K$ under which that state $\mathbf{x}_{0}$ is Neglect Benevolent. Thus, for any exponentially stable linear system, when the set of human inputs $\mathcal{U}_{h}$ is non-empty, the set of of Neglect Benevolent states $\mathcal{X}_{n b}$ is non-empty. Therefore, any exponentially stable linear system exhibits Neglect Benevolence.

\section{Robot Swarm Configuration Control}

In this section, we use the above quantitative approach to analyze Neglect Benevolence in human control of robot swarm configurations. We first design a control law for a 
robot swarm system so it can form various desired spatial configurations. The swarm system under the designed control law has a symmetric dynamics matrix, and we apply the analysis results presented in the previous section to optimize the configuration control of the system.

Consider a swarm of robots where each robot has a bidirectional communication link with some subset of the other robots in the swarm. Assume that the communication topology of robots can be modeled by an undirected connected graph $\mathcal{G}=(\mathcal{V}, \mathcal{E})$, where each vertex is a robot and each edge is a bidirectional communication link. The adjacency matrix for the graph is given by $\mathbf{A}_{\text {adjacency }}=$ $\left[a_{i j}\right]$, where $a_{i i}=0$ and $a_{i j} \geq 0$ is the weight of the edge between vertex $i$ and vertex $j$ in the graph. If such an edge does not exist in the graph, $a_{i j}=0$. Note that in an undirected graph, the adjacency matrix is symmetric. The Laplacian matrix is given by $\mathbf{L}=\left[l_{i j}\right]$, where $l_{i i}=\sum_{j} a_{i j}$ and $l_{i j}=-a_{i j}$. Note that

$$
[1,1, \ldots, 1] \mathbf{L}=\mathbf{L}[1,1, \ldots, 1]^{T}=\mathbf{0}
$$

As presented in [29], the following continuous time control law can be applied at every robot:

$$
\dot{\mathbf{x}}(t)=-\mathbf{L x}(t)
$$

where the vector $\mathbf{x}(t)$ represents the state of the system at time $t$, and is a concatenation of all the state values $x_{i}(t)$ of each robot $i$ in the system. Under the control law, the system would converge to a state $\mathbf{x}_{\text {final }}$.

$$
\mathbf{x}_{\text {final }}=\frac{\sum_{i} x_{i}(0)}{n}[1,1, \ldots, 1]^{T}
$$

Each robot has the same state value, which is the average of all robots' initial states. If the state of each robot is multidimensional (e.g. x-positions, y-positions) then Equation 17 holds for each dimension. This means that if the state represents the spatial location of robots, then all robots would rendezvous under the control law.

Below we design another control law for a connected robot swarm system, so that all robots would rendezvous but in a desired spatial formation around the centroid of their initial location, specified beforehand by a vector parameter $K$.

Lemma 1: Under the following control law:

$$
\dot{\mathbf{x}}(t)=-\mathbf{L}(\mathbf{x}(t)-K)
$$

the robot swarm state would converge to $\mathbf{x}_{\text {final }}$, with individual robot's state difference specified by $K$ (i.e. the robot swarm would rendezvous to a desired spatial formation specified by $K$ ), around the initial centroid.

Proof: First, we show that the sum of all robots' state values is always a constant.

$$
\begin{aligned}
\sum_{i=1}^{n} \dot{x}_{i}(t) & =[1,1, \ldots, 1] \dot{\mathbf{x}}(t) \\
& =-[1,1, \ldots, 1] \mathbf{L}(\mathbf{x}(t)-K)=0 \\
& \sum_{i=1}^{n} x_{\text {final }, i}=\sum_{i=1}^{n} x_{i}(0)
\end{aligned}
$$

where $x_{\text {final, } i}$ represents the final state of robot $i$.

Next we show that when the state of all robots converges, the difference among their state values is specified by $K$.

$$
\dot{\mathbf{x}}_{\text {final }}=0 \Rightarrow \mathbf{L}\left(\mathbf{x}_{\text {final }}-K\right)=0
$$

Since we assume that $\mathcal{G}$ is connected, the eigenvector of $\mathbf{L}$ corresponding to eigenvalue 0 is $a[1,1, \ldots, 1]^{T}$, where $a \in \mathbb{R}$.

$$
\mathbf{x}_{\text {final }}=K+a[1,1, \ldots, 1]^{T}
$$

Combining Equation (20) and (22), we have that

$$
x_{\text {final }, i}=\frac{\sum_{i} x_{i}(0)}{n}+\left(K_{i}-\frac{\sum_{i} K_{i}}{n}\right)
$$

So the centroid of the convergence state is the same as the initial centroid, but robots have a spatial formation, specified by $K$.

Now if we consider the situation when the human operator intends to give a new input $K_{2}$ after the initial $K_{1}$, depending on the input time $t_{\text {input }}$ of $K_{2}$, the control law is

$$
\dot{\mathbf{x}}(t)=-\mathbf{L}\left(\mathbf{x}(t)-K_{1}+\left(K_{1}-K_{2}\right) \theta\left(t-t_{\text {input }}\right)\right)
$$

We want to impose the new input $K_{2}$ at an optimal time $t_{\text {input }}^{*}$ so that the transient time to the formation specified by $K_{2}$ is minimized. Since the dynamics matrix $-\mathbf{L}$ is symmetric, it must be normal. So the system can be viewed as a special case of systems discussed in Section IV-A. So we can apply the results in Section IV-A to analyze Neglect Benevolence for robot swarm configuration control.

\section{RESUltS FOR RobOt SWARM CONFIGURATION CONTROL}

The method of generating various robot configurations presented in the previous section can be used to generate rigid formations for the robots as shown in Figure 2. If the state vector $\mathrm{x}$ represents the concatenated $\mathrm{x}$-positions and y-positions of the robots, the vector $K$ specifies a spatial distribution for the robots. As the robots are moving into a natural goal formation under the influence of the system dynamics, the human may become aware of a new goal to be achieved with the swarm prior to a fixed deadline. The human would then want to provide an input to influence the swarm into a different formation required to achieve the new goal prior to the deadline. In this case, the performance criterion comes in two parts: (1) the swarm must achieve the desired formation before the deadline and (2) minimizing the total time required to achieve the goal formation is desirable. To meet the deadline and minimize the time required to achieve the goal, the human may naively try to provide input as soon as possible (i.e. immediately after becoming aware of the new goal). However, the theory of Neglect Benevolence developed in previous sections indicates that there may exist certain states of the system in which applying the input immediately causes the system to have worse performance than delaying the application of input. Specifically, delaying the application of input may increase system performance. 


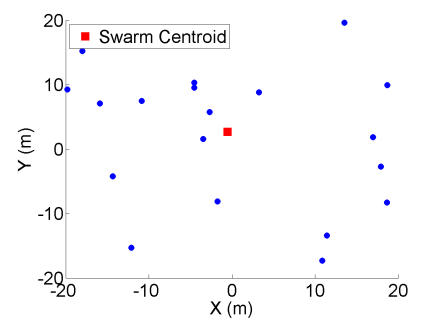

(a) Initial Positions of the Robots

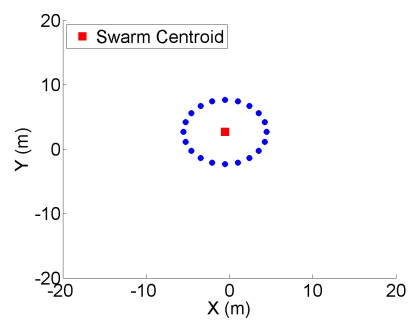

(b) Robot Formation Achieved when No Human Input is Applied

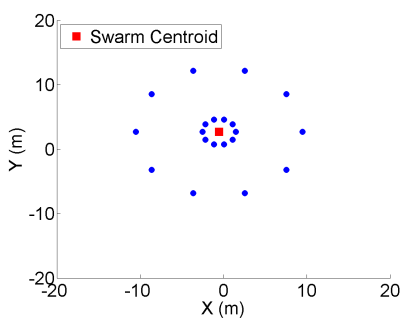

(c) Robot Formation Desired by the Human and Generated when Human Input is Applied

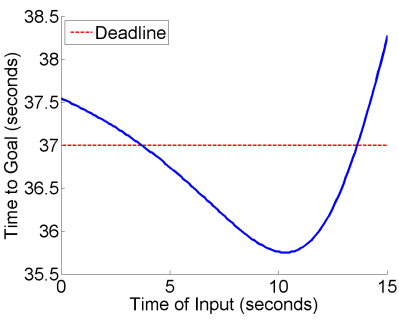

(d) Time at which the Desired Formation is Achieved vs. Time at which Human Input is Applied

Fig. 2: Neglect Benevolence in Controlled Rendezvous Formation

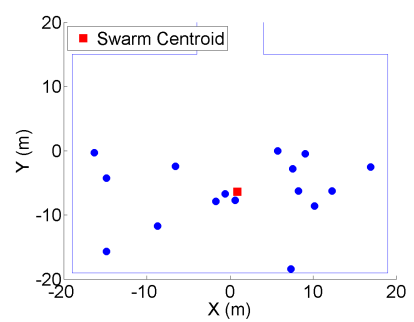

(a) Initial Positions of the Robots

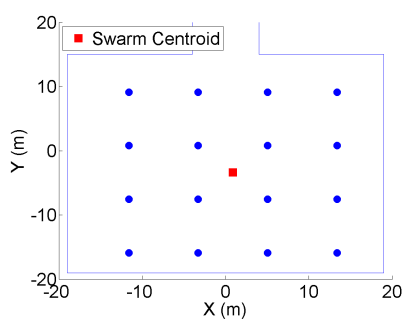

(b) Robot Formation Achieved when No Human Input is Applied

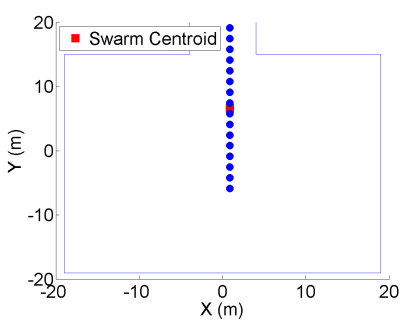

(c) Robot Formation Desired by the Human and Generated when Human Input is Applied

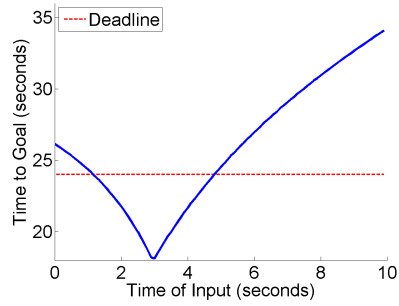

(d) Time at which the Desired Formation is Achieved vs. Time at which Human Input is Applied

Fig. 3: Neglect Benevolence when Moving Robots into a Narrow Passage

Several simulations demonstrated that this system for generating robot configurations did indeed exhibit Neglect Benevolence. Simulated swarms consisted of between 3 and 25 holonomic robots that could communicate bidirectionally with a fixed subset of other swarm members (i.e. communication graph not assumed to be complete). Each simulated robot could measure only its own position on the map.

Results from one such simulation are shown in Figure 2. In Figure 2a, the robots are shown initially dispersed across the map. If no human input is provided to the swarm, the system dynamics naturally drive the robots into the circular formation shown in Figure 2b. However, when the swarm is in the initial state shown in Figure 2a, the human becomes aware of a new goal that requires the swarm to be in the torus formation shown in Figure 2c. The human also becomes aware that the swarm must achieve this formation within 37 seconds and that it is preferable to achieve the formation as early as possible. As shown in Figure 2d, if the human naively tells the swarm to form a torus immediately at $t=0$, the swarm does not achieve the formation within the minimum amount of time. In fact, the swarm does not achieve the torus formation prior to the deadline at all, much less in the minimum amount of time! The human must wait approximately 4 seconds before influencing the swarm in order for the swarm to create a torus formation before the deadline. If the human delays the application of input by approximately 10 seconds, then the swarm achieves the torus formation within the minimum amount of time.

Figure 3 presents simulation results from another scenario that also requires the swarm to achieve different formations and exhibits Neglect Benevolence. In this simulation, the swarm begins in a room in the initial configuration shown in Figure 3a. It is assumed that prior to the start of the simulation the human operator's primary goal was to maximize exploration of the room and the human chose to do so by informing the swarm to form a grid. If no further human influence is provided, the swarm will naturally move into the grid formation shown in Figure 3b. However, immediately after the human operator has applied the input for forming the grid configuration, they are given a new goal that requires moving the swarm into the narrow passage. To achieve this goal, the human informs the swarm to move toward the passage. This input appears as a constant bias term in the system dynamics equation and causes the swarm centroid to move towards the passage. To avoid colling with the walls of the room before reaching the passage, the human must also inform the swarm to create the line formation shown in Figure 3c. The first robots in the swarm will reach the passage (or collide into the walls) at 24 seconds, but this implicit deadline is not necessarily known to the human. In the previous section, it was shown that the system dynamics will always ensure the formation is centered on the (in this case, moving) centroid of the swarm. As shown in Figure $3 \mathrm{~d}$, if the human applies the input immediately, the swarm will not achieve the formation before reaching the narrow passage. There is a narrow window of opportunity between approximately 1 and 5 seconds when applying the input enables the swarm to achieve the new formation prior to 
reaching the passage. In addition, there is an optimal input time at 3 seconds, when applying the input will enable the swarm to move into the line formation by 18 seconds, well before the 24 second implicit deadline.

\section{CONCLUSion AND Future Work}

Robotic swarms that autonomously coordinate via simple local control laws are becoming increasingly interesting for applications such as reconnaissance, surveillance and disaster response. These applications are characterized by uncertainty and potential changes of the environment and mission goals. This results in the human redirecting the swarm by changing its goals and behaviors. In prior experimental work in Human Swarm Interaction, we observed that system performance often depended on the timing of the operator input to the swarm. In particular, we observed that in many instances, system performance improved if the operator, instead of giving input to the system immediately as soon as mission goals changed, delayed giving the new input to the system. We labeled this notion of beneficial delay as Neglect Benevolence. In this paper, we formalized this new notion and presented ways to quantify this notion as well as procedures to compute the optimal time for the operator to give input. We also proved the existence of Neglect Benevolence for a class of linear systems. Experimental simulation results showed that in many situations where the system must reach a specified human goal within some deadline, if the human does not consider the effects of Neglect Benevolence in timing her input, the system may never achieve the goal. This paper is the first to formally introduce the notion of Neglect Benevolence and present initial results. There remain many additional challenges and areas of research that we will consider in future work. Some of the issues we plan to address are: (a) obtain further formal results for a sequence of goals and new human inputs, (b) prove the existence of Neglect Benevolence for different measures of system performance (e.g. energy minimization), (c) provide characterization of Neglect Benevolence and optimal timing of human input for non-linear systems, (d) investigate system sensitivity to the phenomenon of Neglect Benevolence with regard to noise in sensor measurements or state estimates.

\section{ACKNOWLEDGMENTS}

This research has been sponsored in part by ONR Grant N0001409-10680.

\section{REFERENCES}

[1] A. Kolling, S. Nunnally, and M. Lewis, "Towards human control of robot swarms," in Proceedings of the 7th international conference on Human-robot interaction. ACM, 2012.

[2] S. Bashyal and G. Venayagamoorthy, "Human swarm interaction for radiation source search and localization," in IEEE Swarm Intelligence Symposium. IEEE, 2008, pp. 1-8.

[3] P. Walker, S. Nunnally, M. Lewis, A. Kolling, N. Chakraborty, and K. Sycara, "Neglect benevolence in human control of swarms in the presence of latency," in IEEE International Conference on Systems, Man, and Cybernetics, 2012, pp. 3009-3014.

[4] V. Gazi and K. M. Passino, "Stability analysis of social foraging swarms," IEEE Trans. on Systems, Man and Cybernetics, vol. 34, pp. 539-557, 2004.
[5] C. W. Reynolds, "Flocks, herds, and schools: A distributed behavioral model," in SIGGRAPH '87, 1987, pp. 25-34.

[6] I. D. COUZIN, J. KRAUSE, R. JAMES, G. D. RUXTON, and N. R. FRANKS, "Collective memory and spatial sorting in animal groups," Journal of Theoretical Biology, vol. 218, no. 1, pp. 1-11, 2002.

[7] W. Spears and D. Spears, Physicomimetics: Physics-Based Swarm Intelligence. Springer-Verlag New York Inc, 2012.

[8] D. Bruemmer, "A robotic swarm for spill finding and perimeter formation," DTIC Document, Tech. Rep., 2002.

[9] F. Bullo, J. Cortés, and S. Martínez, Distributed Control of Robotic Networks. Princeton University Press, 2009.

[10] H. Choset, "Coverage for robotics-a survey of recent results," Annals of Mathematics and Artificial Intelligence, vol. 31, no. 1, pp. 113-126, 2001.

[11] F. Ducatelle, G. A. Di Caro, and L. M. Gambardella, "Cooperative self-organization in a heterogeneous swarm robotic system," in Proceedings of the 12th annual conference on Genetic and evolutionary computation, 2010, pp. 87-94.

[12] T. Balch and R. Arkin, "Behavior-based formation control for multirobot teams," Robotics and Automation, IEEE Transactions on, vol. 14, no. 6, pp. 926-939, 1998.

[13] M. Turpin, N. Michael, and V. Kumar, "Decentralized formation control with variable shapes for aerial robots," in IEEE Int. Conf. on Robotics and Automation, May 2012.

[14] M. Egerstedt and X. Hu, "Formation constrained multi-agent control," Robotics and Automation, IEEE Transactions on, vol. 17, no. 6, pp. 947-951, 2001.

[15] R. Beard, J. Lawton, and F. Hadaegh, "A coordination architecture for spacecraft formation control," Control Systems Technology, IEEE Transactions on, vol. 9, no. 6, pp. 777-790, 2001.

[16] M. Cummings, "Human supervisory control of swarming networks," in 2nd Annual Swarming: Autonomous Intelligent Networked Systems Conference, 2004.

[17] G. Coppin and F. Legras, "Autonomy spectrum and performance perception issues in swarm supervisory control," Proceedings of the IEEE, no. 99, pp. 590-603, 2012.

[18] Z. Kira and M. Potter, "Exerting human control over decentralized robot swarms," in 4th International Conference on Autonomous Robots and Agents, 2009, pp. 566-571.

[19] M. Fields, E. Haas, S. Hill, C. Stachowiak, and L. Barnes, "Effective robot team control methodologies for battlefield applications," in IEEE/RSJ International Conference on Intelligent Robots and Systems, 2009 , pp. $5862-5867$.

[20] M. Goodrich, B. Pendleton, P. Sujit, and J. Pinto, "Toward human interaction with bio-inspired robot teams," in IEEE International Conference on Systems, Man, and Cybernetics, 2011, pp. 2859-2864.

[21] J. Crandall and M. Goodrich, "Characterizing efficiency of human robot interaction: a case study of shared-control teleoperation," in IEEE/RSJ International Conference on Intelligent Robots and Systems, vol. 2, 2002, pp. 1290-1295 vol.2.

[22] D. R. Olsen, Jr. and S. B. Wood, "Fan-out: measuring human control of multiple robots," in Proceedings of the SIGCHI Conference on Human Factors in Computing Systems, 2004, pp. 231-238.

[23] D. Olsen and M. Goodrich, "Metrics for evaluating human-robot interactions," in Proceedings of PERMIS, vol. 2003, 2003.

[24] A. Steinfeld, T. Fong, D. Kaber, M. Lewis, J. Scholtz, A. Schultz, and M. Goodrich, "Common metrics for human-robot interaction," in Proceedings of the 1st ACM SIGCHI/SIGART conference on Humanrobot interaction, 2006, pp. 33-40.

[25] P. Mitchell and M. Cummings, "Management of multiple dynamic human supervisory control tasks," in 10th International Command and Control Research and Technology Symposium, 2005.

[26] S. Mau and J. Dolan, "Scheduling to minimize downtime in humanmultirobot supervisory control," 2006.

[27] Y. Xu, T. Dai, K. Sycara, and M. Lewis, "Service level differentiation in multi-robot teams," in IEEE/RSJ International Conference on Intelligent Robots and Systems, October 2010, pp. 2224-2230.

[28] S.-Y. Chien, M. Lewis, S. Mehrotra, N. Brooks, and K. Sycara, "Scheduling operator attention for multi-robot control," in IEEE/RSJ International Conference on Intelligent Robots and Systems, 2012, pp. 473-479.

[29] W. Ren, R. Beard, and E. Atkins, "A survey of consensus problems in multi-agent coordination," in Proceedings of the 2005 American Control Conference, 2005, pp. 1859-1864. 